Int. J. Morphol.,

39(1):167-171, 2021

\title{
Susceptibilidad de las Mucosas Oculares al SARS COV-2: Bioseguridad Sanitaria
}

\author{
Susceptibility of Eye Mucosa to SARS CoV-2: Sanitary Biosecurity
}

Héctor Rodríguez Bustos ${ }^{1}$; Monserrat Vásquez Rojas²; Patricio Gutiérrez Morales²; Yessenia Vargas Lara²; Paola Vieytas ${ }^{2}$; Camilo Arriaza Onel ${ }^{1}$ \& Omar Espinoza-Navarro $^{3}$

\begin{abstract}
RODRÍGUEZ, B. H.; VÁSQUEZ, R. M.; GUTIÉRREZ, M. P.; VARGAS, L. Y.; VIEYTAS, P.; ARRIAZA, O. C. \& ESPINOZANAVARRO, O. Susceptibilidad de las mucosas oculares al SARS COV-2: Bioseguridad sanitaria. Int. J. Morphol., 39(1):167-171, 2021.

RESUMEN: El SARS CoV-2,agente causal de la enfermedad llamada Covid-19, infecta las mucosas digestivas y respiratorias, afectando las células epiteliales. El virus ingresa a través del receptor de membrana ACE2 provocando la disrupción de la homeostasis celular. Frecuentes reportes indican la presencia de conjuntivitis ocular en pacientes diagnosticadas con Covid-19, lo cual ha alertado a los científicos sobre el potencial foco de infección viral de las secresiones lagrimales.Los epitelios de la conjuntiva ocular sub-palpebral y corneal, se caracterizan por presentar el receptor de la enzima convertidora de angiotensina 2 (ACE2) y proteasa transmembrana asociada serina 2 (TMPRSS2), cuya interacción activa los mecanismos de liberación de citoquinas, capaces de instalar un proceso de conjuntivitis infecciosa por SARS CoV-2, pero no necesariamente hacer extensiva la infección hacia los sistemas digestivo y respiratorio.Aunque este proceso inflamatorio es más frecuente como una expresión de la infección general y más grave. Sin embargo, cualquiera sea la vía de infección o ingreso del virus SARS CoV-2 es importante considerar el riesgo de infectividad de las lágrimas y las secresiones conjuntivales en los pacientes. Este estudio pretende llamar la atención sobre las medidas de cuidados y control sanitario, incorporando mejores normas de protección personal y bioseguridad, especialmente en el áreas de oftalmología, asumiendo que la mucosa ocular puede ser una vía de entrada del virus y a la vez una fuente de contagio. También considerar la potenciación de la infección viral con las enfermedades de base asociadas, como glaucoma y diabetes.Se sugiere además incorporar estudios histológicos de la mucosa ocular para diferenciar epitelios sanos e infectados.
\end{abstract}

PALABRAS CLAVE: Epitelio Ocular; Covid-19; Bioseguridad Sanitaria; Glaucoma; Oftalmología.

\section{INTRODUCCIÓN}

El 11 de febrero de 2020 la OMS nombró oficialmente SARS-CoV-2 al virus que provoca la enfermedad del COVID-19 (Organización Mundial de la Salud, 2020a), el 11 de marzo 2020 se declara pandemia global, dada la alta propagación del virus a nivel mundial (Organización Mundial de la Salud, 2020b). La enfermedad Covid-19 corresponde a una enfermedad de declaración obligatoria (EDO) o enfermedad de notificación obligatoria (ENO), lo cual significa que está incluida en el grupo de enfermedades trasmisibles que los profesionales de la salud están obligados a notificar al centro de salud pública respectivo, por ser de riesgo para la comunidad (Ministerio de Salud, 2020). Según Guo et al. (2020), desde el coronavirus del síndrome respiratorio agudo severo (SARS-CoV) en 2002 y luego en Oriente Medio (2012), con el coronavirus del síndrome respiratorio (MERS-CoV), la aparición de SARS-CoV-2, mar- ca la tercera introducción de un coronavirus altamente patógeno, originando una pandemia a gran escala en la población humana. Los síntomas clínicos de los pacientes con COVID-19 incluyen fiebre, tos, fatiga y síntomas de infección gastrointestinal. Los ancianos y las personas con enfermedades subyacentes corresponden a la población más susceptibles a la infectabilidad.

COVID-19 afecta principalmente al sistema respiratorio, siendo su acción mayor sobre el epitelio alveolar (epitelio simple plano). La lesión de las células epiteliales alveolares sería la principal causa del síndrome de dificultad respiratoria aguda (Li \& Ma, 2020). Sin embargo Wei et al. (2020), advierten que la infección puede transmitirse a través de múltiples rutas. Kaur et al. (2020) mencionan la presencia de una gran cantidad de citoquinas/quimioquinas

\footnotetext{
${ }^{1}$ Morphology, Instituto de Ciencias Biomédicas, ICBM. Faculty of Medicine, University of Chile, Chile.

${ }^{2}$ Laboratorio de Morfología, Facultad de Medicina, Universidad de Atacama, Chile.

${ }^{3}$ Departamento de Biología, Facultad de Ciencias, Universidad de Tarapacá, Chile.
} 
proinflamatorias que serían los responsables de la gravedad de la enfermedad, más aún cuando hay otras enfermedades coexistentes como diabetes e hipertensión y hábito como el consumo de tabaco.

Kwok et al. (2015) describen que las medidas epidemiológicas más importantes para prevenir los contagios y difusión de las enfermedades contagiosas son el uso de mascarillas, el lavado frecuente de mano y el distanciamiento físico (distancia entre personas). Sin embargo, los ojos y la mucosa conjuntival no han recibido mayor atención como una potencial ruta relevante de infección e ingreso del virus a las vías respiratorias. Hu et al. (2020), determinaron que la falta de protección ocular fue el factor de riesgo principal en la transmisión del SARS-CoV, desde los pacientes, a los trabajadores de la salud,en el año 2003, en Toronto, Canadá. Lo que generó la preocupación de que las enfermedades respiratorias pudieran transmitirse a través de las secreciones oculares.Por lo tanto, resulta relevante analizar la literatura existente sobre la importancia de la conjuntiva ocular en esta infección viral. Más aún cuando se usan distinta técnicas para la identificación del virus y sus vías de infección. La recolección de la información se realizó ocupando la plataforma de Informática del Sistema Universitario de Chile, con acceso directo y completo a la PubMed y Medline.Las láminas histológicas analizadas, corresponden al repositorio docente de la Facultad de Medicina de la Universidad de Chile.

Ludwig et al. (2020) determinan que para entender las enfermedades y afecciones que se pueden presentar en los ojos, conviene entender la anatomía y fisiología básica del ojo.Estructuralmente el globo ocular incluye partes expuestas del ojo como la conjuntiva palpebral que recubre internamente ambos párpados y la conjuntiva ocular que recubre externamente la córnea (iris y pupila). Por estar en un ambiente húmedo la conjuntiva ocular pasa a conformar una mucosa, que incluye el epitelio yel tejido conectivo subyacente. La mucosa conjuntival está formada por un epitelio estratificado plano no cornificado que recubre toda la superficie expuesta húmeda del ojo, que cuando se dispone sobre la región de la córnea se denomina epitelio anterior de la córnea y le subyace la membrana de Bowman. La exposición al medio externo de la córnea podría actuar como potencial receptor de la infección viral (Fig.1). Histológicamente la córnea se continúa con la esclerótica y entre ambas estructuras se forma el conducto esclerocorneal revestido por un delgado endotelio y que drena los fluidos de las cámaras del ojo, que también sería potencial lugar de inflamación en los procesos de conjuntivitis (Fig. 2). Un estudio realizado por Loffredo et al. (2020), informan que la conjuntivitis también llamada "ojo rosa", es una enfermedad frecuente de etiología bacteriana y viral y se ha descrito asociada a infec- ción por coronavirus, provocando inflamación y vasculitis. Zhang et al. (2020) sugieren que el nuevo coronavirus puede infectar y reinfectar el tracto respiratorio a través de la conjuntiva como una potencial ruta de infección desde el saco conjuntival y las células acinares de la glándula lagrimal, conducto naso lagrimal hacia la cavidad nasal, a través de la presencia del receptor ACE2.

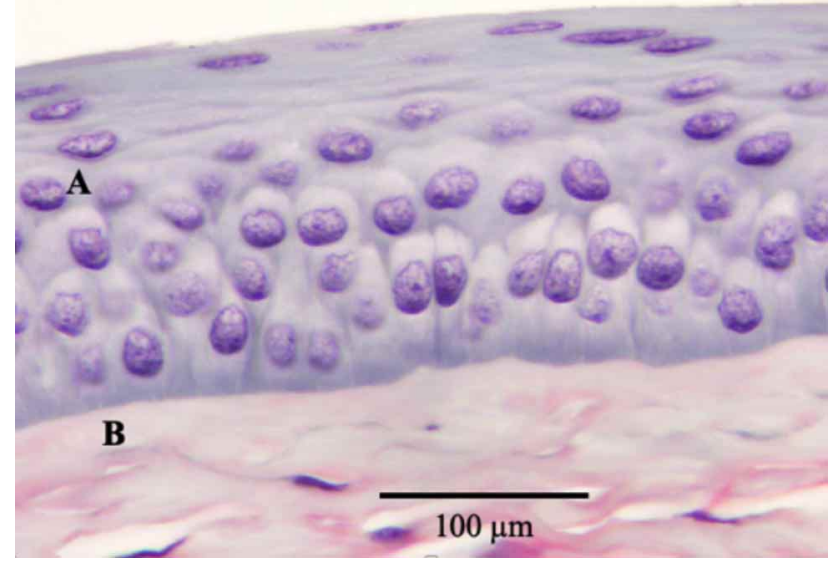

Fig. 1. Microfotografía de la córnea del ojo humano. En A se observa el epitelio estratificado plano de la córnea anterior, en B se observa la membrana de adhesión conectiva al estroma.Tinción H\&E 100x.

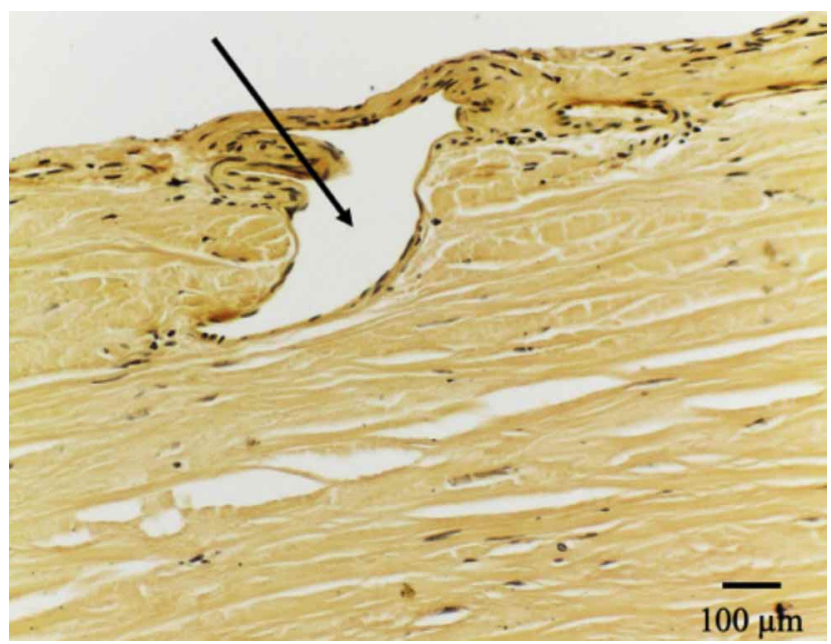

Fig. 2. Microfotografía de la pared anterior-lateral del ojo humano. La flecha muestra el canal esclero-corneal de drenaje, revestido de un endotelio delgado. Tinción Argéntica, 40x.

Holappa et al. (2015) describen que la enzima convertidora de angiotensina (ACE2), juegaun papel importante en el sistema renina-angiotensina (SRA), tomando un rol beneficioso en muchas enfermedades como hipertensión, diabetes y enfermedades cardiovasculares donde su expresión está disminuida. Ragia \& Manolopoulos (2020) y Napoli et al. (2020), describen que el ACE2 y la serina proteasa transmembrana 2 (TMPRSS2), son elementos proteicos esenciales para la interacción del SARS CoV-2 en las célu- 
las huésped. Por lo que todas las células que lo expresan son una potencial ruta de ingreso del virus. El receptor ACE2 se expresa normalmente en una gran cantidad de tipos celulares diferentes, Zhou et al. (2020) describen que en las muestras de conjuntiva, limbo y córnea (post mortem y biopsias quirúrgicas) de pacientes confirmados con Covid19 , se presenta una fuerte reacción positiva al reconocimiento tisular de ACE2 y TMPRSS2. Sin embargo, Lange et al. (2020) no observan expresión del receptor ACE2, ni de sus cofactores funcionales a través de técnicas de PCR en la conjuntiva sana. Ma et al. (2020) describen que solo en algunos tipos celulares conjuntivales hay expresión génica para ACE2, al igual que la expresión del cofactor TMPRSS2. Seah et al. (2020), Wu et al. (2020) y Torres-Costas et al. (2020) encuentran muy baja positividad viral en muestras oculares (lágrimas) en pacientes confirmados de Covid-19, lo que estaría indicando que la vía ocular conjuntival no siempre participa de la infección, aunquesi podría asociarse con pacientes más graves. Hong et al. (2020) destacan que los pacientes más graves de Covid-19 tienen más probabilidades de presentar sintomatología ocular incluso justo antes de la aparición de los síntomas respiratorios (prodrómicos). Lange et al. concluyen que a pesar de la baja expresión de ACE2 y sus cofactores en la conjuntiva, esta podría ser una ruta de ingreso de la infección hacia el sistema respiratorio, concluyendo que la vía ocular sí es una vía de infección, aunque de baja ocurrencia. Según Güemes-Villahoz et al. (2020), la presencia de ARN del SARS-CoV-2 en muestras oculares destaca el papel del ojo como posible vía de transmisión de la enfermedad. Colavita et al. (2020), en estudios en hospitales italianos, plantean que la afectación ocular viral, podría ocurrir al comienzo de la infección Covid-19 y que el virus puede replicarse en la conjuntiva y representar una fuente potencial de infectividad. La fisiopatología exacta de la transmisión ocular del virus sigue sin comprenderse por completo, aunque existe evidencia que el SARS-CoV-2 se detecta en las secreciones oculares por lo tanto vale la pena considerar el tropismo ocular del virus y su potencial para causar enfermedad ocular localizada (Ho et al, 2020).

Gegúndez-Fernández et al. (2020) y Rousseau et al. (2020) en conjunto con las Sociedades Españolas y Francesas de Oftalmología promueven la adopción de medidas de acción y protección para la atención oftalmológica en consultas externas, áreas quirúrgicas y hospitalización, para pacientes COVID-19.La información obtenida de estudios en China y Europa muestra que la edad avanzada, género masculino, ser portador de enfermedades crónicas (cardiovascular, respiratoria, diabetes, cáncer), tabaquismo e inmunosupresión son factores de peor pronóstico. La diabetes afecta la integridad de la superficie ocular y aumenta la susceptibilidad a infecciones oculares a nivel ocular pu- diendo favorecer la infectabilidad con SARS-CoV-2. El glaucoma es una patología donde la edad es un factor de riesgo preponderante y que en la gran mayoría de los casos tiene una evolución lenta. Los pacientes de más edad son a su vez los más frágiles frente al Covid-19 (Bertoli et al., 2020).

Para evitar una infección cruzada, las sociedades de Oftalmología y de Glaucoma sugieren reagendar las consultas que no son urgentes durante esta pandemia, evitar realizar oftalmoscopía directa y en todas las consultas implementar el uso de mascarilla y protección ocular (lentes, gafas, antiparras, mascarilla con protección ocular).La Sociedad Chilena de Oftalmología, recomienda la creación de redes de apoyo para la atención remota de estos pacientes de riesgo (Lai et al., 2020; Napoli et al.; Sociedad Chilena de Oftalmología, 2020). Una reflexión final es recordar que el primer médico en informar sobre esta nueva enfermedad fue el Dr. Li Wenliang, médico oftalmólogo, quien murió después de padecer la enfermedad. Esto ejemplificaría las implicaciones de los oftalmólogos en pandemias de enfermedades infecciosas (Bacherini et al., 2020).

\section{CONCLUSIONES}

El epitelio de la conjuntiva ocular sub-palpebral y corneal, se caracterizan por presentar el receptor ACE2 y las proteínas de membrana asociadas(TMPRSS2), cuya interacción activa los mecanismos de liberación de citoquinas, capaces de instalar un proceso de conjuntivitis infecciosa por SARS CoV-2.La literatura publicada en tiempos de pandemia hace referencia que pacientes con Covid19, cursan problemas oculares variables tipo conjuntivitis. Por lo tanto independiente de las medidas de cuidados y control de lavado frecuente de manos, no tocarse la cara, nariz, ojos y el uso de mascarillas, se debe incorporar mejores normas de protección personal asumiendo que la mucosa ocular puede ser una vía de entrada del virus y a la vez una fuente de contagio, considerando la potenciación de la infección viral con las enfermedades oculares de base asociadas, como diabetes y glaucoma. Se suguiere además, incorporar análisis histológicos, en los estudios para diferenciar epitelios sanos, de infectados con Covid-19.

\section{AGRADECIMIENTOS}

Este trabajo contó con el auspicio del proyecto UTA Mayor N 4716-17, Universidad de Tarapacá, AricaChile. 
RODRÍGUEZ, B. H.; VÁSQUEZ, R. M.; GUTIÉRREZ, M. P.; VARGAS, L. Y.; VIEYTAS, P.; ARRIAZA, O. C. \& ESPINOZA-NAVARRO, O. Susceptibility of eye mucosa to SARS CoV-2: Sanitary biosecurity. Int. J. Morphol., 39(1):167$171,2021$.

SUMMARY: SARS CoV-2, the causal agent of the Covid19 disease, infects the digestive and respiratory mucosa, affecting epithelial cells. The virus enters through the ACE2 membrane receptor causing the disruption of cell homeostasis. Frequent reports indicate the presence of ocular conjunctivitis in patients diagnosed with Covid-19, which has alerted scientists to the potential source of viral infection from lacrimal secretions. The epithelia of the subpalpebral and corneal ocular conjunctiva are characterized by presenting the receptor for angiotensin-converting enzyme 2 (ACE2) and associated transmembrane protease serine 2 (TMPRSS2), whose interaction activates cytokine release mechanisms, with the ability to start the infectious conjunctivitis process by SARS CoV-2, but not necessarily extend the infection to the digestive and respiratory systems. Although this inflammatory process is more frequent as an expression of the general and more serious infection. However, whatever the route of infection or entry of the SARS CoV-2 virus, it is important to consider the risk of infection of tears and conjunctival secretions in patients. This study aims to draw attention to health care and control measures, incorporating better standards of personal protection and biosafety, especially in the areas of ophthalmology, assuming that the ocular mucosa can be a route of entry for the virus, and at the same time a source of contagion. A further consideration is the potential of viral infection with associated underlying diseases, such as glaucoma and diabetes. It is also suggested to incorporate histological studies of the ocular mucosa to differentiate healthy and infected epithelia.

KEY WORDS: Ocular Epithelium; Covid-19; Health Biosafety; Glaucoma; Ophthalmology.

\section{REFERENCIAS BIBLIOGRÁFICAS}

Bacherini, D.; Biagini, I.; Lenzetti, C.; Virgili, G.; Rizzo, S. \& Giansanti, F. The COVID-19 pandemic from an ophthalmologist's perspective. Trends Mol. Med., 26(6):529-31, 2020.

Bertoli, F.; Veritti, D.; Danese, C.; Samassa, F.; Sarao, V.; Rassu, N.; Gambato, T. \& Lanzetta, P. Ocular findings in COVID-19 patients: A review of direct manifestations and indirect effects on the eye. $J$. Ophthalmol., 2020:4827304, 2020.

Colavita, F.; Lapa, D.; Carletti, F.; Lalle, E.; Bordi, L.; Marsella, P.; Nicastri, E.; Bevilacqua, N.; Giancola, M. L.; Corpolongo, A.; Ippolito, G.; et al. SARS-CoV-2 isolation from ocular secretions of a patient with COVID-19 in Italy with prolonged viral RNA detection. Ann. Intern. Med., 173(3):242-3, 2020.

Gegúndez-Fernández, J. A.; Zarranz-Ventura, J.; Garay-Aramburu, G.; Múñoz-Negrete, F. J.; Mendicute del Barrio, J.; Pablo-Júlvez, L.; García-Delpech, S.; López-Alemany, A.; Arnalich-Montiel, F.; Cordero-Coma, M.; et al. Recommendations for eye care during the alarm state by the coronavirus disease pandemic COVID-19. Arch. Soc. Esp. Oftalmol., 95(6):300-10, 2020.

Güemes-Villahoz, N.; Burgos-Blasco, B.; Arribi-Vilela, A.; ArriolaVillalobos, P.; Rico-Luna, C. M.; Cuiña-Sardiña, R.; Delgado-
Iribarren, A. \& García-Feijoó, J. Detecting SARS-CoV-2 RNA in conjunctival secretions: Is it a valuable diagnostic method of COVID-19? J. Med. Virol., 2020. DOI: https://www.doi.org/ 10.1002/jmv.26219

Guo, Y. R.; Cao, Q. D.; Hong, Z. S.; Tan, Y. Y.; Chen, S. D.; Jin, H. J.; Tan, K. S.; Wang, D. Y., \& Yan, Y. The origin, transmission and clinical therapies on coronavirus disease 2019 (COVID-19) outbreak - an update on the status. Mil. Med. Res., 7(1):11, 2020.

Ho, D.; Low, R.; Tong, L.; Gupta, V.; Veeraraghavan, A. \& Agrawal, R. COVID-19 and the ocular surface: a review of transmission and manifestations. Ocul. Immunol. Inflamm., 28(5):726-34, 2020.

Holappa, M.; Valjakka, J. \& Vaajanen, A. Angiotensin(1-7) and ACE2, "the hot spots" of renin-angiotensin system, detected in the human aqueous humor. Open Ophthalmol. J., 9:28-32, 2015.

Hong, N.; Yu, W.; Xia, J.; Shen, Y.; Yap, M. \& Han, W. Evaluation of ocular symptoms and tropism of SARS-CoV-2 in patients confirmed with COVID-19. Acta Ophthalmol., 2020. DOI: https:// www.doi.org/10.1111/aos.14445

Hu, K.; Patel, J. \& Patel, B. C. Ophthalmic Manifestations of Coronavirus (COVID-19). Treasure Island (FL), Stat Pearls Publishing, 2020.

Kaur, G.; Lungarella, G. \& Rahman, I. SARS-CoV-2 COVID-19 susceptibility and lung inflammatory storm by smoking and vaping. J. Inflamm. (Lond.), 17:21, 2020.

Kwok, Y. L. A.; Gralton, J. \& McLaws, M. L. Face touching: a frequent habit that has implications for hand hygiene. Am. J. Infect. Control, 43(2):112-4, 2015.

Lai, T. H. T.; Tang, E W. H..; Chau, S. K. Y.; Fung, K. S. C. \& Li, K. K. W. Stepping up infection control measures in ophthalmology during the novel coronavirus outbreak: an experience from Hong Kong. Graefe's Arch. Clin. Exp. Ophthalmol., 258(5):1049-55, 2020.

Lange, C.; Wolf, J.; Auw-Haedrich, C.; Schlecht, A.; Boneva, S.; Lapp, T.; Horres, R.; Agostini, H.; Martin, G.; Reinhard, T.; et al. Expression of the COVID-19 receptor ACE2 in the human conjunctiva. J. Med. Virol., 2020. DOI: https://www.doi.org/10.1002/ jmv. 25981

Loffredo, L.; Pacella, F.; Pacella, E.; Tiscione, G.; Oliva, A. \& Violi, F. Conjunctivitis and COVID-19: A meta-analysis. J. Med. Virol., 2020. DOI: https://www.doi.org/10.1002/jmv.25938

Ludwig, P. E.; Jessu, R. \& Czyz, C. N. Physiology, Eye. Treasure Island (FL), StatPearls Publishing, 2020.

Ma, D.; Chen, C. B.; Jhanji, V.; Xu, C.; Yuan, X. L.; Liang, J. J.; Huang, Y.; Cen, L. P. \& Ng, T. K. Expression of SARS-CoV-2 receptor ACE2 and TMPRSS2 in human primary conjunctival and pterygium cell lines and in mouse cornea. Eye (Lond.), 34(7):1212-9, 2020.

Ministerio de Salud (MINSAL). Informe Epidemiológico Enfermedad por SARS-CoV-2. (Covid-19). 13-04-2020. Santiago de Chile, Ministerio de Salud, Gobierno de Chile, 2020.

Napoli, P. E.; Nioi, M.; d'Aloja, E. \& Fossarello, M. The ocular surface and the Coronavirus Disease 2019: Does a dual 'ocular route' exist? J. Clin. Med., 9(5):1269, 2020.

Organización Mundial de la Salud (OMS). Naming the Coronavirus Disease (COVID-19) and the Virus that Causes It. Ginebra, Organización Mundial de la Salud, 2020a. Disponible en: https:// www.who.int/emergencies/diseases/novel-coronavirus-2019/ technical-guidance/naming-the-coronavirus-disease-(covid-2019)and-the-virus-that-causes-it

Organización Mundial de la Salud (OMS). Novel Coronavirus(2019$n C o V)$. Situation Report - 11. Ginebra, Organización Mundial de la Salud, 2020b. Disponible en: https://www.who.int/docs/defaultsource/coronaviruse/situation-reports/20200131-sitrep-11ncov.pdf?sfvrsn=de7c0f7_4 
Ragia, G. \& Manolopoulos, V. G. Inhibition of SARS-CoV-2 entry through the ACE2/TMPRSS2 pathway: a promising approach for uncovering early COVID-19 drug therapies. Eur. J. Clin. Pharmacol., 2020. DOI: https://www.doi.org/10.1007/s00228-02002963-4

Rousseau, A.; Fenolland, J. R. \& Labetoulle, M. SARS-CoV-2, COVID19 and the eye: An update on published data. J. Fr. Ophtalmol., 43(7):642-52, 2020.

Seah, I. Y. J.; Anderson, D. E.; Kang, A. E. Z.; Wang, L.; Rao, P.; Young, B. E.; Lye, D. C. \& Agrawal, R. Assessing viral shedding and infectivity of tears in Coronavirus Disease 2019 (COVID-19) patients. Ophthalmology, 127(7):977-9, 2020.

Sociedad Chilena de Oftalmología (SOCHIOF). Recomendaciones Generales de la Sociedad Chilena de Oftalmología para Minimizar el Riesgo de Infección por Covid-19 en Nuestros Centros de Salud. Comunicado 2020. Santiago de Chile, Sociedad Chilena de Oftalmología, 2020. Disponible en: https://www.sochiof.cl/dinamicos/ documentos/COVID-19recomendaciones-generales-final.pdf

Torres-Costa, S.; Lima-Fontes, M.; Falcão-Reis, F. \& Falcão, M. SARSCOV-2 in ophthalmology: Current evidence and standards for clinical practice. Acta. Med. Port., 33(9):593-600, 2020.

Wu, P.; Duan, F.; Luo, C.; Liu, Q.; Qu, X.; Liang, L. \& Wu, K. Characteristics of ocular findings of patients with Coronavirus Disease 2019 (COVID-19) in Hubei Province, China. JAMA Ophthalmol., 138(5):575-8, 2020.

Zhang, B. N.; Wang, Q.; Liu, T.; Dou, S. Q.; Qi, X.; Jiang, H.; Qi, B. X.; Zhang, B. \& Zhou, Q. J. A special on epidemic prevention and control: analysis on expression of 2019-nCoV related ACE2 and TMPRSS2 in eye tissues. Zhonghua Yan Ke Za Zhi, 56(6):438-46, 2020.

Zhou, L.; Xu, Z.; Castiglione, G. M.; Soiberman, U. S.; Eberhart, C. G. \& Duh, E. J. ACE2 and TMPRSS2 are expressed on the human ocular surface, suggesting susceptibility to SARS-CoV-2 infection. Ocular Surf., 18(4):537-44, 2020.

\author{
Dirección para correspondencia: \\ Omar Espinoza-Navarro \\ Departamento de Biología \\ Facultad de Ciencias \\ Universidad de Tarapacá \\ Arica \\ CHILE
}

Email: oespinoz@academicos.uta.cl

Recibido: 24-10-2020

Aceptado: 09-11-2020 